\title{
Be stars: one ring to rule them all?
}

\author{
A. Meilland ${ }^{1}$, Ph. Stee ${ }^{1}$, J. Zorec ${ }^{2}$, and S. Kanaan ${ }^{1}$ \\ 1 Observatoire de la Côte d'Azur, CNRS-UMR 6203, Avenue Copernic, Grasse, France \\ e-mail: anthony.meilland@obs-azur.fr \\ 2 Institut d'Astrophysique de Paris, UMR7095 CNRS, Univ. P. \& M. Curie, Paris, France
}

Received 14 December 2005 / Accepted 2 March 2006

\begin{abstract}
Aims. We report the theoretical spectral energy distributions (SEDs), Br $\gamma$ line profiles, and visibilities for two scenarios that can explain the disk dissipation of active hot stars and account for the transition from the Be to the B spectroscopic phase.

Methods. We use the SIMECA code to investigate two scenarios, the first one where the disk is formed by successive outbursts of the central star. A low-density region is developing above the star and slowly grows outward and forms a ring-like structure that will gradually excavate the disk. The second one has a slowly decreasing mass loss due for instance, to a decrease in the radiative force through an opacity change at the base of the photosphere, and may also be responsible for the vanishing of the circumstellar disk.

Results. We find that a clear signature of the disk dissipation following the ring scenario will be the disappearance of the high velocity tails in the emission lines and a nearly constant peak separation. Moreover, we found that, following the ring-like scenario, the visibilities must show an increasing second lobe, an increase in the value of the first zero, and assuming an unresolved central star, a first zero of the visibility curves that appends at shorter baselines as far as the disk has been excavated. We propose to use the AMBER instrument on the VLTI to probe whether the ring scenario is the one to rule the Be phenomenon.
\end{abstract}

Key words. techniques: high angular resolution - techniques: interferometric - stars: emission-line, Be - stars: winds, outflows circumstellar matter

\section{Introduction}

According to advances in the study of stellar mass-loss phenomena since the discovery of Be stars, different kinds of models have attempted to describe their circumstellar envelope (CE) or disk formation. Although they coexist, we may attempt to divide the mass-ejection phenomena into two broad classes: 1) continuing mass ejections and 2) discrete mass ejections. Even though one or the other of these mass-loss events might be prevalent, somewhat different types of CE structures could be envisioned:

1) CONTINUING MASS EJECTIONS. The most frequently suggested mechanisms of $\mathrm{CE}$ formation evoke continuing mass ejections. Struve $(1931,1942)$ imagined Be stars as secularly stable critical rotators that build a rotating extended envelope flattened towards the equator through equatorial ejection of mass (Limber 1964, 1967, 1969). Somewhat modified by the inclusion of an expansion velocity field, these CE structures were used by Marlborough (1969) and Poeckert \& Marlborough (1987a,b) to predict observable spectroscopic, photometric, and polarimetric quantities. Thenceforth, several ad hoc flaring and flattened disc-shaped empirical CE models were explored in the literature to see if the CEs were outwardly accelerated, decelerated, and/or rotationally supported (Waters 1986; Waters et al. 1987; Kastner \& Mazzali 1989; Hummel \& Dachs 1992; Hummel 1994; Hanuschik 1995, 1996; Wood et al. 1997). Thomas \& Doazan (1982) proposed an active spherical exophotospheric structural pattern, where the low-density outwardly accelerated hot chromospheric-coronal zone is followed by a cool and decelerated high density region. However, interferometric and polarimetric observations, as well as statistical inferences from spectroscopy, support some flattening of CE in Be stars
(Arias et al. 2006; Quirrenbach et al. 1997; Tycner et al. 2005). In the frame of theoretical inferences of CE properties based on continuing mass ejections, the thinness of circumstellar discs in Be stars still remains an open issue (Araújo \& Freitas Pacheco 1989; Bjorkman \& Cassinelli 1993; Chen et al. 1992; Owocki et al. 1996; Poe \& Friend 1986; Porter 1997; Stee \& Araújo 1994; Stee et al. 1995, 1998). Moreover, from physical first principles (Schmitz 1983) and the kinetic theory of gases applied to steady-state isothermal self-gravitating gaseous masses with cylindrical differential rotation, Rohrmann (1997) predicts bagel-shaped CE that are not reminiscent of flat discs.

2) DISCRETE MASS EJECTIONS. In addition to these continuing mass ejections, there are discrete mass ejections. They can be divided roughly into two types: 1) small-scale to moderate ejections, detected either as sharp absorption features crossing the line profiles or as a sudden appearance of emission shoulders in the wings of some absorption lines and/or $\mathrm{H} \alpha$ line emission outbursts that imply ejecta with masses $\Delta M \lesssim 10^{-11} M_{\odot}$ (cf. Floquet et al. 2000); 2) Large-scale or massive ejections. A clear example is $\omega$ Ori, where a sudden light increase of nearly $\Delta V \approx-0.3 \mathrm{mag}$ was reported by Guinan \& Hayes (1984). The polarimetric data and $\mathrm{H} \alpha$ spectroscopy of the event were interpreted by Brown \& Wood (1992) as due to a discrete mass ejection equivalent to an instantaneous mass-loss rate $\dot{M} \approx$ $2.5 \times 10^{-8} M_{\odot} \mathrm{yr}^{-1}$. The sudden $\mathrm{H} \alpha$ emission outbursts in $\mu$ Cen on timescales ranging from 20 to 245 days were interpreted by Hanuschik et al. (1993) as being produced by discrete mass ejecta of $\Delta M \approx 10^{-10} M_{\odot}$. In the past decade, short- and longlived photometric outbursts have been discovered (Cook et al. 1995; Hubert \& Floquet 1998; Keller et al. 2002; Mennickent et al. 2002) from MACHO, OGLE, and HIPPARCOS data. They are 
currently called "flickers" or "sharp" variations when the time scales of phenomena range from $t \sim 50$ to 200 days, and they imply light changes of $\Delta V \lesssim 0.2 \mathrm{mag}$. They are called "bumpers" or "humps" if $t \gtrsim 200$ days to 3-4 years and $\Delta V \gtrsim 0.3 \mathrm{mag}$. Mennickent et al. (2002) speculate that thermal instabilities may produce these photometric behaviors, while Moujtahid et al. (1999), Hubert et al. (2000), and Zorec et al. (2000a) have attempted to explain them in terms of sudden or discrete massejections of $\Delta M \approx 10^{-10} M_{\odot}$. These variations also summarize the irregular photometric behaviors, including the well-known $\mathrm{B} \rightleftharpoons \mathrm{Be} \rightleftharpoons \mathrm{Be}$-shell phase transitions, observed in the past more or less sporadically (cf. Pavlovski et al. 1997; Percy et al. 1997; Moujtahid et al. 1998). Finally, we should not omit mentioning the far-UV C IV, Si III, and Si IV discrete line-absorption components in stars with $V \sin i \gtrsim 150 \mathrm{~km} \mathrm{~s}^{-1}$ (Grady et al. 1987, 1989), which were interpreted as due to wind instabilities, but may actually correspond to discrete mass-ejections.

According to these observational grounds for mass ejections, two scenarios can be sketched to roughly account for the $\mathrm{CE}$ variations and/or formation, to which the observed long-term Be star emission variations should be associated.

This paper is organized as follows. In Sect. 2 we introduce the two possible scenarios for the mass ejection. In Sect. 3 we briefly present the SIMECA code and its main assumptions. Section 4 presents the ring scenario and Sect. 5 treat the vanishing mass flux scenario. The comparison of different observables (photometric, spectroscopic, and interferometric) is done in Sect. 6. Finally, in Sect. 7 we summarize and discuss our main results.

\section{Two possible scenarios for the mass ejection}

\section{SCENARIO 1: EXPANDING RINGS}

The stellar wind interacts with the ISM and the matter strewn by earlier mass-loss events to create a propagating "shell"-front. If we neglect details for shocks, compression, heating, and cooling phenomena in the wind+close interstellar interaction region (Pikel'ner 1986; Kwok et al. 1978), the balance between the original stellar wind momentum against that of the wind+swept ISM and spread material gives the phase with mass-flux $\dot{M}_{*}$ (Zorec 1981) for the location of the "shell"-front at the time $t$ since the star began:

$R / R_{\mathrm{O}}=\left[\left(V_{*} / v-1\right)\left(N_{\mathrm{o}} / N_{\mathrm{ISM}}\left(3 V_{*} t / R_{\mathrm{o}}\right)\right]^{1 / 3}\right.$,

where $R_{\mathrm{O}}$ is the radius at which the wind has maximum velocity $V_{*}$, and the particle concentration is $N_{\mathrm{o}} ; v$ is the expansion of the shell'-front; $N_{\text {ISM }} \approx 1 \mathrm{~cm}^{-1}$ is the density in the ISM. The lower limit on $R / R_{\mathrm{O}}$ is set by $V_{*} t=R$. Then $R / R_{\mathrm{O}} \approx 10^{4}$ for a B2e star, taking $R_{\mathrm{O}} \approx 3 R_{*}$ with $R_{*} \approx 6 R_{\odot}, \dot{M}_{*} \approx 10^{-10} M_{\odot} \mathrm{yr}^{-1}$ (Snow $1982), V_{*} \approx 500 \mathrm{~km} \mathrm{~s}^{-1}, v / V_{*} \approx 0.1$. Such a "shell", occurring at $3 \times 10^{4} R_{*}$ after 8 years of steady mass-flow, can hardly satisfy the characteristics required by CEs in Be stars. However, the mass-loss phenomenon is variable. Zorec (1981) suggested then that long-lasting low mass-loss episodes can build up moving bubbles around the star, which may act as dams to pile up matter ejected during later short high mass-loss events and thus increase the density around the star up to values typical of CE. To this view Rivinius et al. (2001) have joined a roughly similar picture, which suggested the appearance of a growing cavity between the star and an already existing CE, which is replenished later on with the mass of new ejecta. According to these suggestions, the variable emission intensity in Be stars might then correspond to the appearance of a density void-like region around the star and an expanding ring-like structure.
This intuitive phenomenological picture can be justified on physical grounds taking both the large-scale discrete ejections and winds into account. As seen above, discrete ejections can be effective providers of mass to form $\mathrm{CE}$ in Be stars. On the other hand, stellar winds can contribute to the global dynamics of the mass stored around the star not only with non negligible amounts of mass, but also with momentum and energy. The CE could then be thought of as an expanding wind-blown bubble formed by the process of interaction between the stellar wind and massive discrete ejecta: discrete ejecta produce a clumpy circumstellar environment, which is then ablated by winds producing a mass-loaded flux. The structure of expanding wind-blown bubbles produced by the interaction of high-speed stellar winds with clumpy environments were studied by Hartquist et al. (1986), Dyson \& Hartquist (1992), and Arthur et al. (1994). According to these authors, the most simplified structure of the circumstellar environment in the stationary snowplow phase encompasses three dynamically distinct regions: 1) a wind expansion region; 2) a decelerated, sub-sonic wind momentum-dominated core; 3) a pressure-dominated super-sonic expanding halo. In the decelerated "core" the density rises with the distance as $R^{4}$ from the radius $R_{\mathrm{m}}$ of minimum environmental density up to a contact radius $R_{\mathrm{c}} \approx\left(2 V_{*} / v_{\mathrm{s}}\right) R_{\mathrm{m}}$ ( $v_{\mathrm{s}}$ is the sound speed) that separates the sonic and super-sonic sides. In the "halo", the density decreases as $\mathrm{e}^{-M^{2} / 2}$ where $M=v / v_{\mathrm{s}}$ is the local Mach number in the flow. According to Arthur et al. $(1994), R_{\mathrm{m}}=\left(3 \dot{M}_{*} / 8 \pi \dot{q}\right)^{1 / 3}$ where $\dot{q}$ is the mass injection rate from the cloud ablation process. To obtain orders of magnitude on the bubble expansion and the amount of mass gathered in it, we integrated the onedimensional rotationless steady-state equations of motion given by Dyson \& Hartquist (1992). Assuming that the ablation of discrete ejecta starts at $R_{\mathrm{m}} \approx R_{*}$ and the expansion of the bubble is dominated by the ablated mass, the time-dependent velocity of the bubble is:

$\dot{R}_{\mathrm{b}} \simeq 0.5\left(V_{*} R_{*}\right)^{1 / 4} t^{1 / 4}$.

Within the same approximation, the gathered mass in the bubble from $R_{\mathrm{m}}$ up to a given external distance $R_{\mathrm{e}} \gg R_{\mathrm{c}}$ scales as:

$M_{\mathrm{CE}} \simeq\left(3 R_{\mathrm{c}} \dot{M}_{*} / V_{*}\right)\left[1+\left(V_{*} / v_{\mathrm{s}}\right)\right]^{2}\left(R_{\mathrm{e}} / R_{\mathrm{c}}\right)^{1.3}$,

where the exponent 1.3 is due to a power-law representation of the density distribution in the halo. With the same parameters as are used to estimate (1) and $R_{\mathrm{e}} \approx 10 R_{*}, T_{\mathrm{e}} \approx 10^{4} \mathrm{~K}$, we find that at $t \sim 1$ day the bubble expands at $\dot{R}_{\mathrm{b}} \approx 180 \mathrm{~km} \mathrm{~s}^{-1}$ and at $\dot{R}_{\mathrm{b}} \approx 14 \mathrm{~km} \mathrm{~s}^{-1}$ for $t \sim 1$ month. In the meantime, the radius of the bubble increases from $R_{\mathrm{b}} \lesssim 4 R_{*}$ to $\gtrsim 8 R_{*}$ and the mass stored in it amounts to some $M_{\mathrm{CE}} \approx 2 \times 10^{-9} M_{\odot}$. In current modelings, the total mass in a $\mathrm{CE}$ with base particle density $N \sim 10^{12} \mathrm{~cm}^{-3}$ ranges from $10^{-10} M_{\odot}$ to $10^{-8} M_{\odot}$, depending on whether the CEs are considered flat disc-shaped or spherical, with uniform density distribution or varying with radius as $\sim R^{-2}$. It is then seen that the wind-discrete ejecta interaction mechanism can store the amount of mass expected in CE of Be stars on short time scales in a ring-like volume around the star. As seen, the expanding ring-like structure leaves a cavity behind it and can act as a dam to reconstruct new mass-enhanced circumstellar environments with matter ejected by later outbursts, as suggested by Zorec (1981) and Rivinius et al. (2001).

\section{ScEnARIO 2: VARIABLE MASS-LOSS RATES}

In the preceding discussion we did not consider the problem of constructing $\mathrm{CE}$ in (or near) Keplerian rotation. Be stars 
are fast, though under-critical, rotators (Frémat et al. 2005). Comparing the angular momentum per unit mass in a disc at Keplerian rotation $J=\left(G M_{*} R\right)^{1 / 2}$ and at a distance $R$ from the star to that at the stellar surface, assumed at critical rotation (Keplerian too $), J_{*}=\left(G M_{*} R_{*}\right)^{1 / 2}$, we see that matter ejected from the star needs at least a factor $J / J_{*}=\left(R / R_{*}\right)^{1 / 2}$ of angular momentum-excess to become Keplerian at $R$. Thus to recover this rotation in the circumstellar disc, Lee et al. (1991), Porter (1999), and Okazaki (2001) envision outward-drifting matter in the circumstellar environment through angular momentum exchange by viscous stress. Unless hydrodynamic or magnetohydrodynamic instabilities in the disc induce variations in its structure, variable emission intensities can be thought of as triggered only by variable mass decretion rates in the present scenario. This viscous decretion disc model seems to describe the time scales of the disc formation correctly, as well as its further dissipation in the Be-shell star $o$ And (Clark et al. 2003). The time scales needed to build up the CE in $\alpha$ Eri, as scaled from the observed long-term $\mathrm{H} \alpha$ line emission increases, also agree with those predicted by this scenario. However, the dissipation of its CE requires somewhat longer time scales than foreseen by this theory (Vinicius et al. 2006).

According to scenarios 1 and 2, the increase in the emission in Be stars should correspond either to the replenishment of a circumstellar cavity by mass input from the star (wind and/or discrete ejecta) or to a continuous decretion rate that is increased at any moment by some mechanism. On the other hand, the fading of emission characteristics would correspond either to a cavity formation according to the ring scenario or to a disc dissipation carried by a stopped or reduced mass-decretion rate.

AIM AND PROGRESSION OF THE PRESENT WORK. With the operational VLTI array, with focal instruments MIDI, and in particular with AMBER, which combines spatial and spectral resolution of $10^{4}$, it is now possible to study the $\operatorname{Br} \gamma$ formation region, as well as the shape and extension of the circumstellar disk with a spatial resolution of few mas; i.e. the angular diameter of 1 mas is for a typical Be star with radius $R_{*}=10 R_{\odot}$ situated at $100 \mathrm{pc}$, while the $\mathrm{Br} \gamma$ formation region extends to some stellar radii. The main purpose of this paper is then to give observational grounds for probing the above scenarios of circumstellar disc evolution by searching whether, through interferometric measurements in the $\mathrm{Br} \gamma$ line, it is possible to distinguish which of both scenarios would better suited to explaining the long-term variation of the emission characteristics in Be stars. We would also like to study the consequences on other observables, such as line profiles and spectral energy distributions (SED) that may carry these scenarios.

In this paper we explore the waning aspect of disks and emissions. Thus, we present simulations of the evolution of a cavity from the inner edge to the outer regions as would happen in Scenario 1 and, on the other hand, calculations of the effects produced by a Be star disk submitted to a decreasing mass-loss rate as suited to Scenario 2. To these purposes, we used the SIMECA code described in previous papers [see Stee \& Araùjo (1994) and Stee \& Bittar (2001)]. In particular, we studied the evolution and variation of the $\mathrm{Br} \gamma$ line profile as a function of time, the changes in the spectral energy distribution (SED) and those of intensity maps with the corresponding visibility curves. As we shall see, the visibility curves are very sensitive to the disk geometry, so that they can probe the dissipation of the circumstellar matter with high accuracy. We can then use them to make a distinction between scenario 1 and 2 .

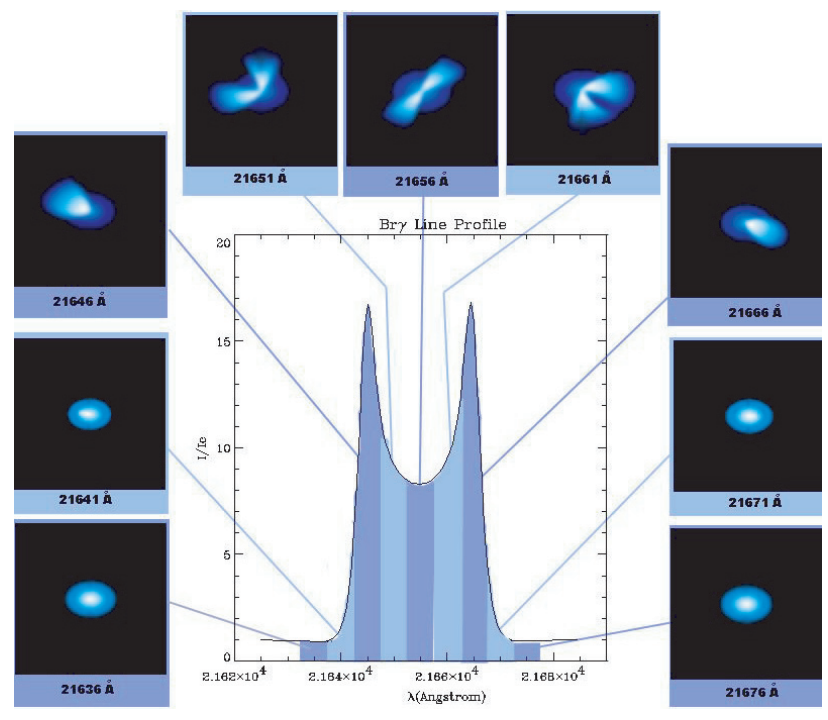

Fig. 1. Isovelocity intensity maps computed with the SIMECA code, seen with an inclination angle of $45^{\circ}$, and showing the effect of the disk kinematics (mainly Keplerian rotation) as a function of wavelength, 21 636, 21 641, 21 646, 21 651, 21 656, 21 661, 21 666, 21 671, and $21676 \AA$ across the Br $\gamma$ line within a spectral bandwidth of $5 \AA$.

\section{SIMECA: a code dedicated to active hot stars}

The SIMECA code computes classical observables, i.e. spectroscopic and photometric ones, but also intensity maps in Balmer lines and in the continuum, in order to obtain theoretical visibility curves that can be directly compared to high angular resolution data. The main hypothesis of this code is that the envelope is axi-symmetric with respect to the rotational axis. No meridian circulation is allowed. We assumed that the physics of the polar regions is represented well by a CAK type stellar wind model (Castor et al. 1975) and the solutions for all stellar latitudes were obtained by introducing a parametrized model that is constrained by spectrally resolved interferometric data. The inner equatorial region is dominated by Keplerian rotation.

The ionization-excitation equations were solved for an envelope modeled in a $411 * 92 * 73$ cube using spherical coordinates, for $r, \theta$ and $\phi$. Since the final population of atomic levels were strongly NLTE distributed, we started with the LTE populations for each level, then computed the escape probability of each transition, which allowed us to obtain updated populations, and we iterated until convergence. The convergence was quite fast (about ten iterations) and stable within an effective temperature of the central star in the range $10000<T_{\text {eff }}<40000$. The basic equations of the SIMECA code are given in detail in Stee \& Araùjo (1994).

To account for the photospheric absorption line, we assumed the underlying star to be a normal B star with a given $T_{\text {eff }}$ and $\log g$. For each $T_{\text {eff }}$ and $\log g$, we computed the $\operatorname{Br} \gamma$ synthetic line profile using the SYNSPEC code developed by Hubeny (Hubeny 1988; Hubeny \& Lanz 1995). These photospheric line profiles are then broadened by solid rotation and can be further absorbed by the envelope volume projected on the stellar disk. The SIMECA code is also able to produce theoretical intensity maps of the circumstellar envelope in this line and in the continuum at different wavelengths. These maps can be directly compared to milli-arcsecond interferometric measurements, such as those obtained from the VLTI interferometer. 

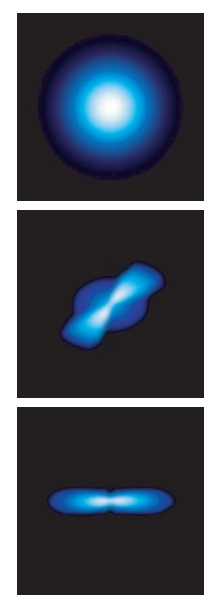
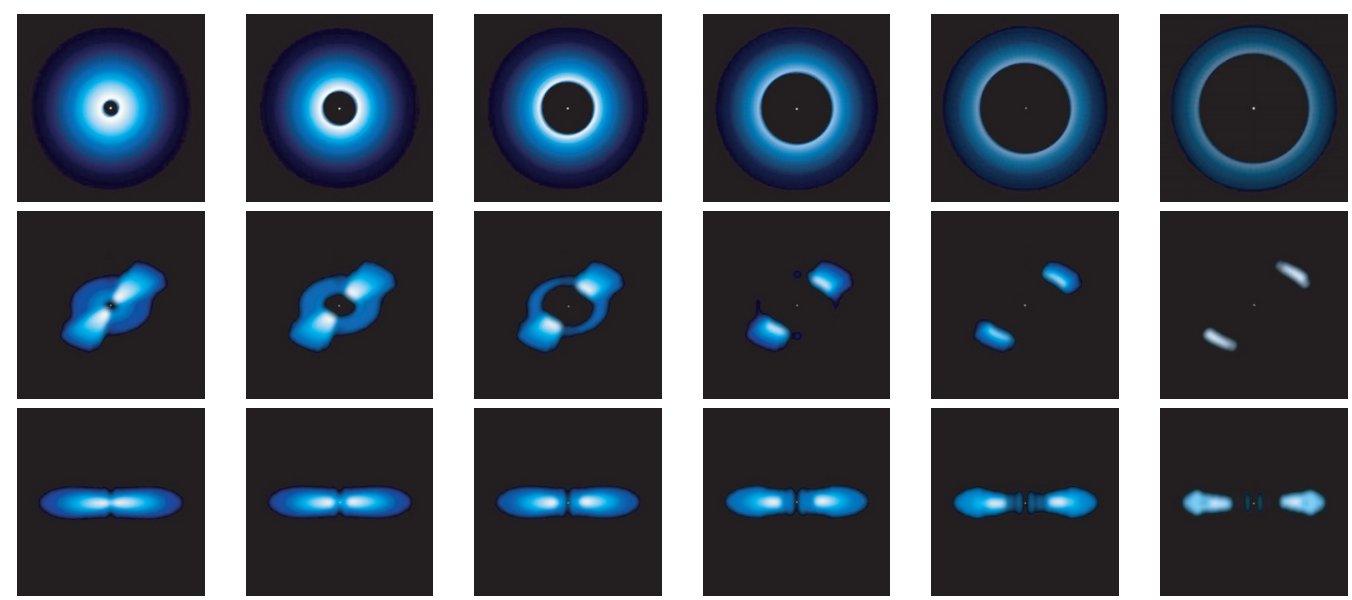

Fig. 2. Intensity maps computed with the SIMECA code showing the formation of a ring ranging from 0 to 60 stellar radii by $10 R_{*}$ steps. Seen pole-on (upper row), at $45^{\circ}$ (center), and equator-on (lower row).
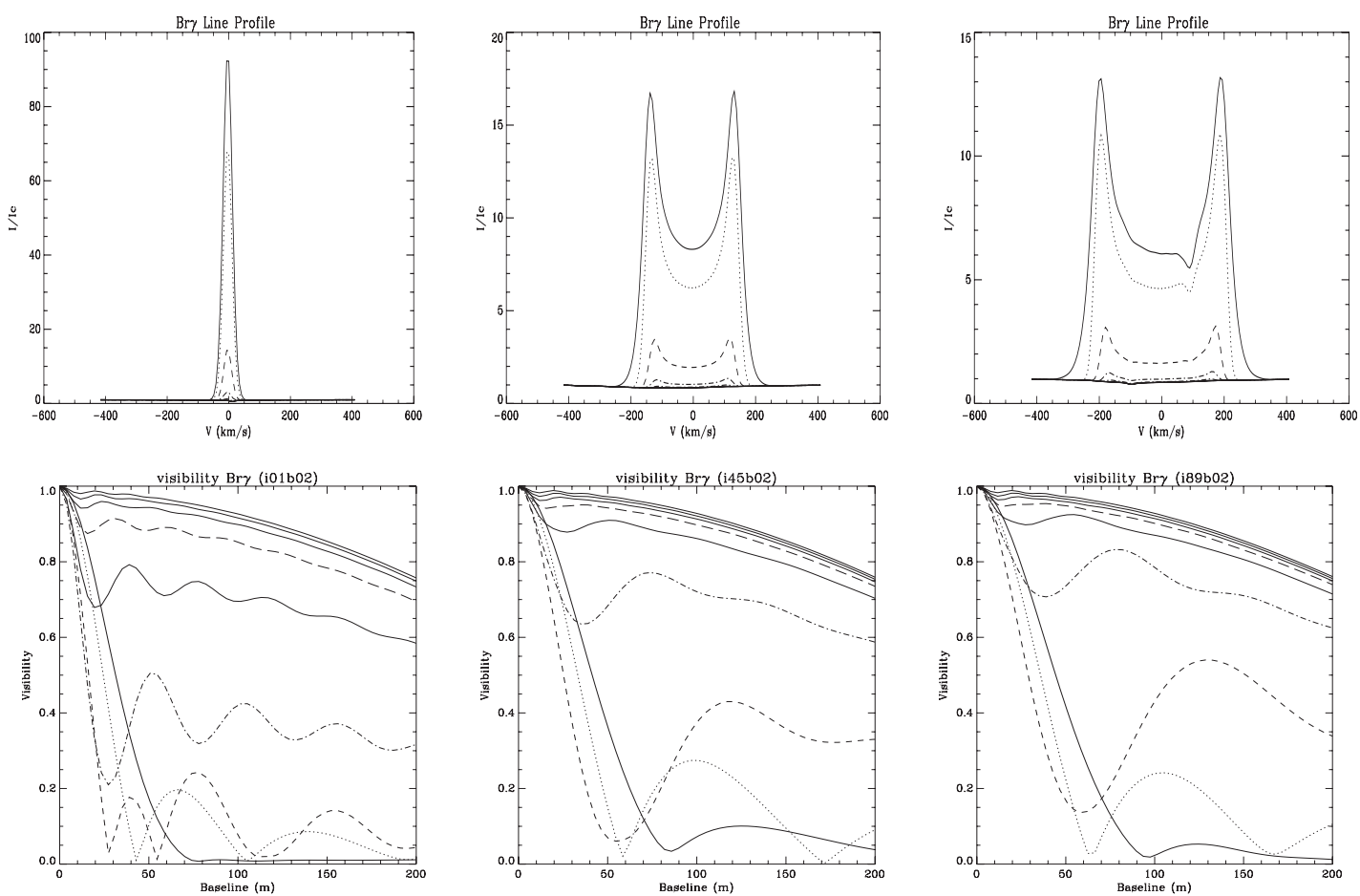

Fig. 3. Br $\gamma$ line profiles (upper line) and visibilities as a function of baseline (lower line) for different ring sizes: $r=0$ (plain line), $r=10$ (dotted line), $r=20$ (dashed line), $r=30$ (dash-dotted line), $r=40$ (dash-dot-dot-dot line), $r=50$ (long-dash line), and for $r=60,70$, and 80 $R_{*}$ (plain line) corresponding to the maps given in Fig. 2. These curves are plotted for 3 inclination angles, i.e. Pole-on (left), $45^{\circ}$ (center), equator-on (right), and for a baseline position along the equatorial disk.

For the $m 1$ parameter, which describes the variation of the mass flux $(\phi)$ from the pole to the equator, we used $m 1=20$ (see Stee 2003, for more details) according to:

$\phi(\theta)=\phi_{\text {pole }}+\left[\left(\phi_{\text {eq. }}-\phi_{\text {pole }}\right) \sin ^{m 1}(\theta)\right]$

where $\theta$ is the stellar colatitude. This $\mathrm{m} 1$ value corresponds to an opening angle of $30^{\circ}$ and thus to a relatively flat disk, as can be seen in the lower row of Figs. 2 and 6. Since the contribution of the polar stellar wind is very faint and the terminal velocity in the dense equatorial region is only $100 \mathrm{~km} \mathrm{~s}^{-1}$, the disk is nearly Keplerian with a rotational velocity of $350 \mathrm{~km} \mathrm{~s}^{-1}$ at the stellar photosphere. It produces a typical double-pics emission line profile with intensity maps that are strongly dependent on the observing wavelength as can be seen in Fig. 1. The basic parameters used to simulate the Be circumstellar disk in the following are given in Table 1.

\section{The ring scenario}

In order to simulate the excavation of the disk, we computed a set of static models where the density is forced to be at a very low value (nearly zero) from the stellar photosphere to a given stellar radius, simulating the evolution of the gradual erosion of the entire disk as can be seen in Fig. 2. Each map is computed at a wavelength centered on the $\mathrm{Br} \gamma$ line within a spectral bandwidth of $5 \AA$ and are plotted for 3 different inclination angles.

The shape of the intensity maps from Fig. 2 ranges from a pure ring-like geometry for the pole-on case to a "bipolar" shape 

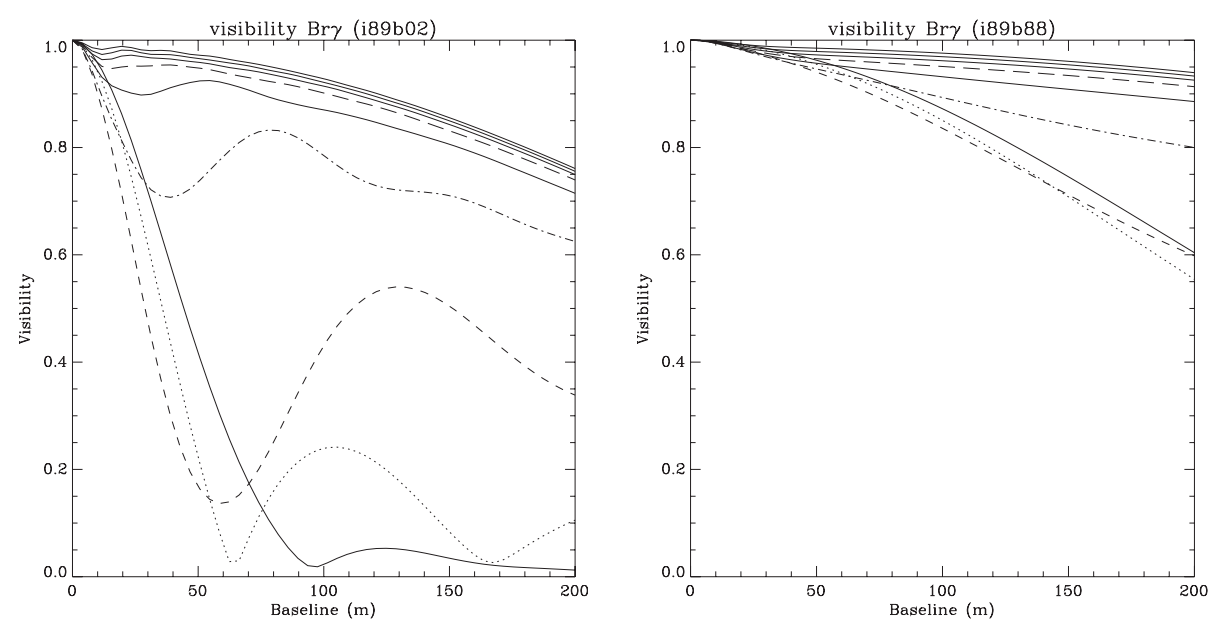

Fig. 4. Visibilities for the ring model as a function of the baseline length for a baseline orientation along (left) and perpendicular (right) to the equatorial disk as a function of the disk dissipation.

Table 1. Parameters and results used in this paper to simulate a Be circumstellar disk.

\begin{tabular}{cc}
\hline \hline$T_{\text {eff }}$ & $22500 \mathrm{~K}$ \\
Radius & $6 R_{\odot}$ \\
Inclination angle $i$ & 0,45 and 90 \\
Photospheric density & $1.0 \times 10^{-12} \mathrm{~g} \mathrm{~cm}^{-3}$ \\
Photospheric expansion velocity & $0.11 \mathrm{~km} \mathrm{~s}^{-1}$ \\
Equatorial rotation velocity & $350 \mathrm{~km} \mathrm{~s}^{-1}$ \\
Equatorial terminal velocity & $100 \mathrm{~km} \mathrm{~s}^{-1}$ \\
Polar terminal velocity & $2000 \mathrm{~km} \mathrm{~s}^{-1}$ \\
Polar mass flux & $4.0 \times 10^{-12} M_{\odot} \mathrm{year}^{-1} \mathrm{sr}^{-1}$ \\
$m 1$ & 20.0 \\
Mass of the disk & $5.06 \times 10^{-10} M_{\odot}$ \\
Mass loss & $4.7 \times 10^{-10} M_{\odot} \mathrm{year}^{-1}$ \\
\hline
\end{tabular}

for the intermediate inclination angle $\left(i=45^{\circ}\right)$ and the equatoron case. The line profiles from Fig. 3 present a single peak for the pole-on case, whereas the increasing inclination angle produces a double-peaked line profile with a larger peak separation for a larger inclination angle. The small absorption close to the center of the $\mathrm{Br} \gamma$ line, on the red side of the line for the edge-on case, is a numerical artifact. In this simulation the assumed central star radius of $6 R_{\odot}$ is placed at a distance of $100 \mathrm{pc}$, and the $\mathrm{Br} \gamma$ circumstellar disk is resolved for baselines as small as 30 meters (for a baseline parallel to the equatorial disk). Of course the disk is not resolved as well if the interferometer baseline is perpendicular to the equatorial disk as shown in Fig. 4. In this latter case the interferometer is not able to "see" the disk dissipation since its angular resolution perpendicular to the equatorial flat disk is not sufficient and the visibilities are similar to those obtained for a uniform disk with a decreasing angular size. The SED curve plotted in Fig. 5 shows the decrease in the IR-excess as a function of the ring extension down to the value where the continuum is only due to the central star (lower plain-line), which is assumed to radiate as a $22500 \mathrm{~K}$ blackbody.

\section{The vanishing mass flux scenario}

In this scenario, the mass loss maybe slowly decreases due to a decrease in the radiative force through an opacity change at the base of the photosphere. In this scenario the mass ejected in the circumstellar disk is becoming smaller and smaller, and

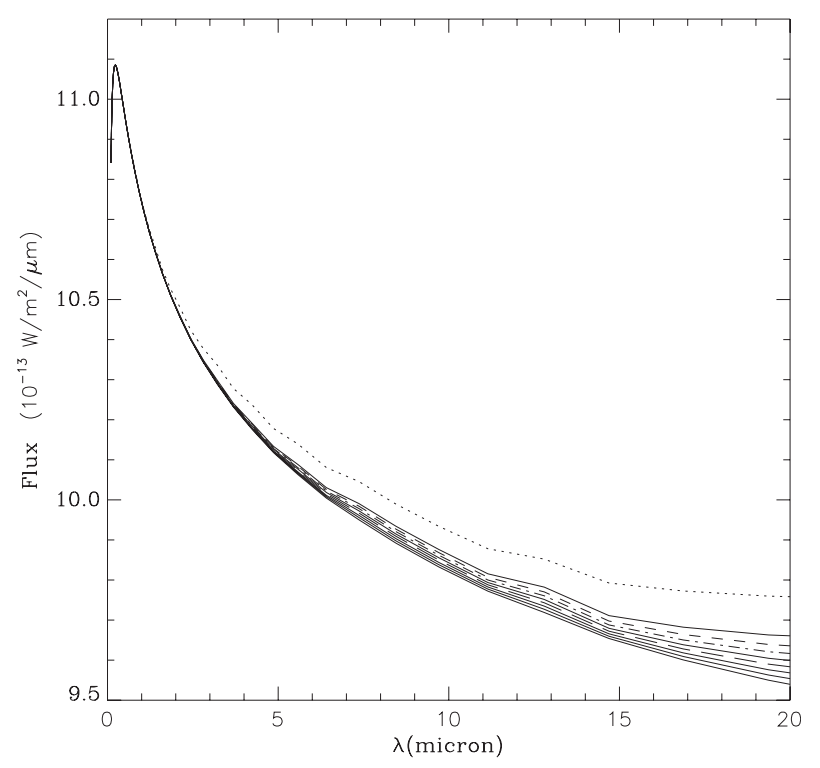

Fig. 5. Spectral energy distribution (SED) as a function of the ring size, for the lower plain line: central star only, $r=0$ (dotted line), $r=10$ (dashed line), $r=20$ (dash-dotted line), $r=30$ (dash-dot-dot-dot line), $r=40$ (long-dash line), and for $r=50,6070$ and 80 (plain line). Since the disk is nearly optically thin the SED is nearly independent of the inclination angle between the star rotation axis and the observer line of sight.

this decreasing brightness contrast between the central star and its surrounding produces a fading of the emitting line profiles (Fig. 7), as well as of the IR excess (Fig. 8). The shape of the $\mathrm{Br} \gamma$ lines is very similar to those obtained for the ring scenario, since the envelope kinematics remains the same for both models. Again, the small absorption close to the center of the $\mathrm{Br} \gamma$ line, on the red side of the line for the edge-on case, is a numerical artifact. Reducing the global mass loss rate by a factor two, namely from $4.7 \times 10^{-10}$ to $2.3 \times 10^{-10} M_{\odot}$ year $^{-1}$ has a strong impact on the intensity of the line profiles, which goes from 95 to 15 times the continuum value for the pole-on case and from 13 to 2 for the equator-on model. This also produces a drastic visibility change, and the disk is fully resolved whatever the inclination angle is with baselines of 70 meters for the pole-on case and 100 meters for the equator-on model for a mass loss rate of 

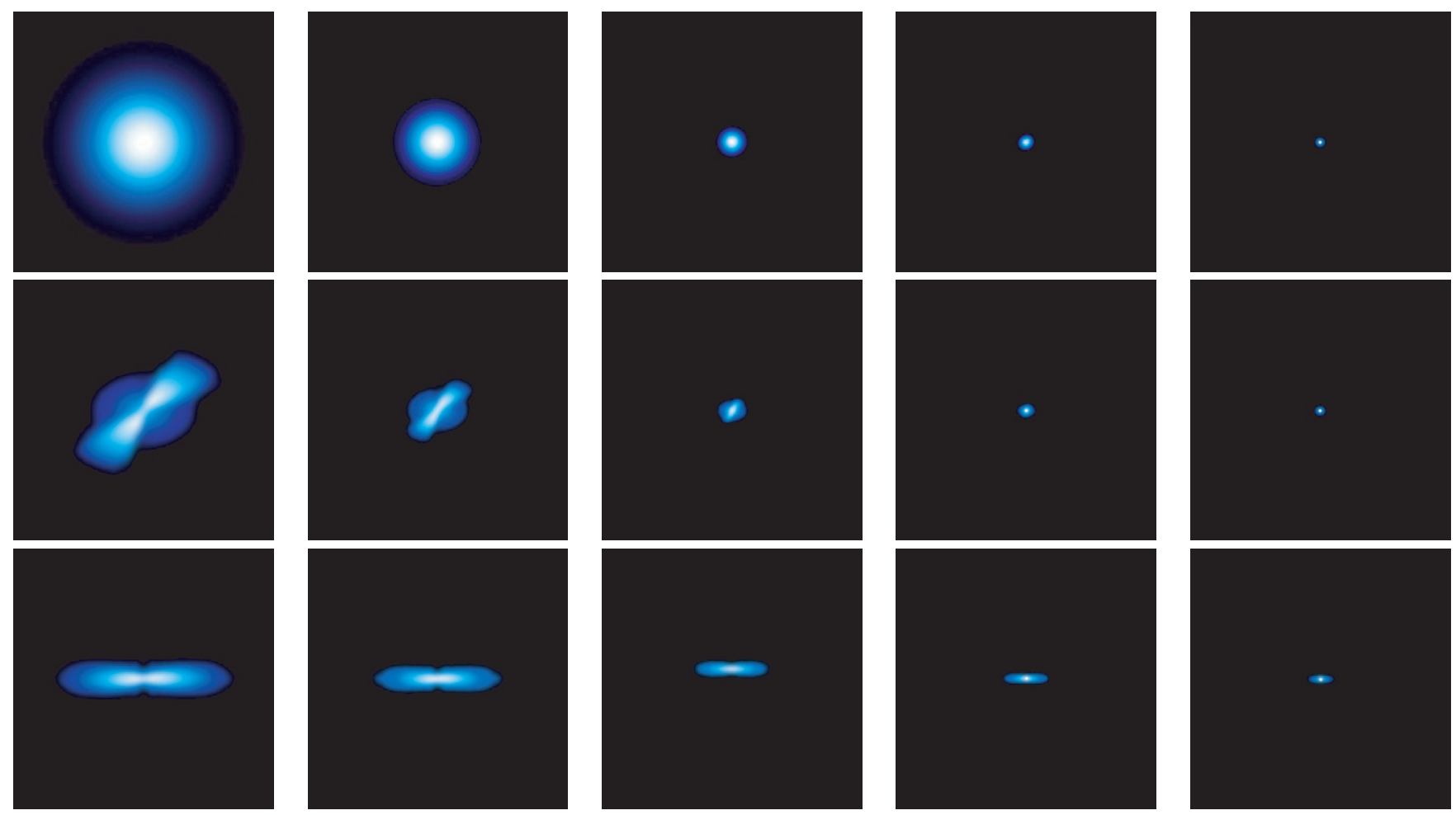

Fig. 6. Intensity maps computed with the SIMECA code showing the vanishing of the circumstellar disk by decreasing the mass flux and thus the global mass loss rates: $4.7 \times 10^{-10}, 2.3 \times 10^{-10}, 9.4 \times 10^{-11}, 4.7 \times 10^{-11}, 2.3 \times 10^{-11} M_{\odot}$ year $^{-1}$ from the left to the right, as seen pole-on (upper row), at $45^{\circ}$ (center), and equator-on (lower row).

$4.7 \times 10^{-10} M_{\odot}$ year $^{-1}$. For the case of the $2.3 \times 10^{-10} M_{\odot}$ year $^{-1}$ mass-loss rate, the visibilities never went to zero for baselines between 0 and 200 meters and the disk appears less resolved for larger inclination angles, i.e. from the pole to the equator. All the visibilities plotted in Fig. 7 are given for an interferometer baseline along the equatorial disk, i.e. horizontal with respect to the intensity maps from Fig. 6.

\section{Comparison of these two scenarios}

Since the aim of this paper was to see if there is a clear difference between the observable (photometric, spectrometric, and interferometric) produced from these two scenarios, we may first compare the SEDs. It is clear from Figs. 5 and 8 that we cannot separate the scenarios: since the envelope is optically, thin there is no difference with respect to the inclination angle; and the continuum produced from a given hydrogen volume is more or less the same, whereas it is distributed in a star-connected envelope or in a circumstellar ring. The differences from the line profiles are more subtle as can be seen in Fig. 9.

In the case of the ring dissipation, the disappearance of the high velocity tails in the emission line is obvious and was already observed by Rivinius et al. (2001) for $28 \mathrm{Cyg}$. This effect is due to the dense Keplerian equatorial disk with a monotonically decreasing rotational velocity. In this case, the emission from the innermost disk region will appear (or disappear) at the outermost line wings. In this paper, we reach the same conclusion as in Rivinus et al. (2001) that the variability in the emission base width is due to density variations in the inner disk. The position of the two line peaks remain mostly unchanged since they originate in the outermost regions of the disk, which have the largest surface area in a given velocity bin. On the other hand, for the other scenario, the diminution of the mass flux produces a global decrease in the line intensity without any noticeable variation in the base line width. Since the line emission is proportional to the volume of the emitting gas, the line intensity decreases more rapidly in this scenario compared to the previous one, as already mentioned in the previous section.

The largest discrepancies come from the visibility curves. From Fig. 10 we can see that the plain line that corresponds to the same starting model, i.e. an extended disk connected to the star surface, shows a fully resolved object with a visibility close to zero for the 100 meters baseline and a very small second lobe of about $5 \%$. Starting with the same model, the differences are straightforward between the model with a ring at $r=10 R_{*}$ and a mass-loss rate of $2.3 \times 10^{-10} M_{\odot}$ year $^{-1}$, i.e. a mass-loss decrease by a factor 2 compared to the previous model. The visibility is resolved for the ring model for a short $65 \mathrm{~m}$ baseline due to the fact that there is less circumstellar matter close to the central star that is also unresolved by the interferometer. Thus, the flux ratio between the unresolved star+inner-envelope and the extended resolved outer-disk decreases and it is easier to "see" this circumstellar outer-matter. This effect also increases the value of the first minimum of the visibility curve which goes from $1 \%$ to $3 \%$ and reaches up to $15 \%$ and $70 \%$ for the $r=20 R_{*}$ and $r=30 R_{*}$ rings, respectively. The second lobe increases as a function of the ring radius up to the case where the flux coming from the ring is small enough to become negligible compared to the stellar flux. In this case the curves converge to a nearly spherical and unresolved object (the central star) that starts to be resolved for baselines on the order of 100-200 m. The shape of the second lobes for the ring model occurs mainly due because we simulate a very sharp inner edge to the disk, i.e. we artificially set the density to zero in the inner ring region. The "true" situation may be less contrasted since the inner edge might be smoother, thereby making a less marked second lobe of the 

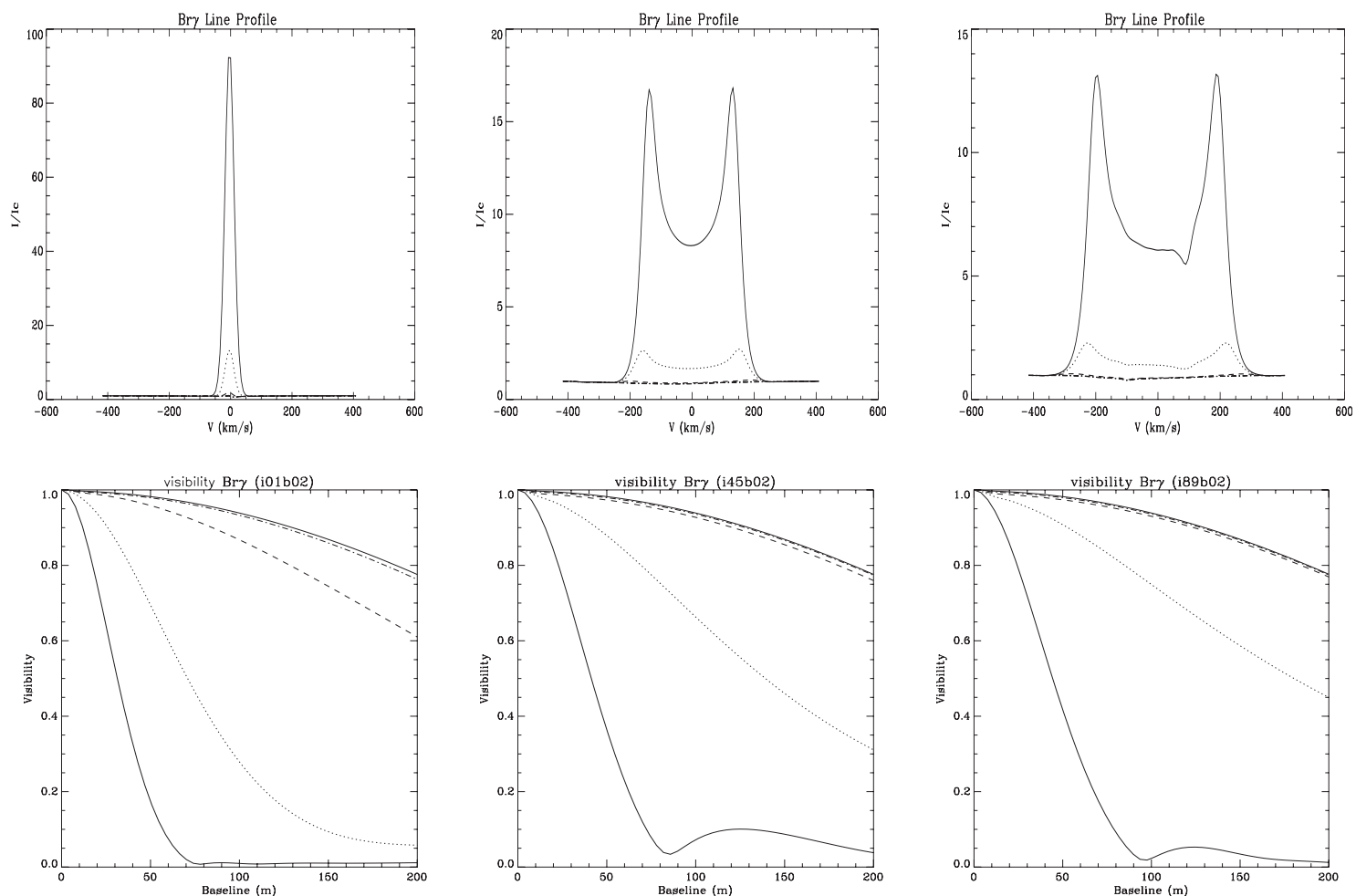

Fig. 7. Br $\gamma$ line profiles (upper row) and visibilities as a function of baseline (lower row) for different mass loss rates, $4.7 \times 10^{-10}$ (plain-line), $2.3 \times 10^{-10}$ (dotted-line), $9.4 \times 10^{-11}$ (dashed-line), and $4.7 \times 10^{-11} M_{\odot}$ year $^{-1}$ corresponding to the maps given in Fig. 6 . These curves are plotted for 3 inclination angles, i.e. Pole-on (left), $45^{\circ}$ (center), equator-on (right) and for a baseline position along the equatorial disk.

visibility. The visibilities for the second scenario are more regular with a global tendency to show a "smooth" object, i.e. a more or less Gaussian brightness distribution and a less and less resolved object. The final shape of the visibilities is similar in both scenarios since the situation converges to the same visibility as the nearly unresolved central star.

\section{Conclusions}

It is clear from this study that, in order to definitely favor the ring scenario of disk dissipation instead of the mass-loss vanishing, we must follow both spectroscopically and interferometrically a Be star that is showing a decrease in its IR excess, as well as in its emission line intensities.

A clear signature of the disk dissipation following the ring scenario could be:

- the disappearance of the high velocity tails in the emission line and a nearly constant peak separation;

- visibilities with an increasing second lobe, an increase in the value of the first zero, and, assuming an unresolved central star, a first zero at shorter baselines as a function of the disk excavation.

Thanks to the VLTI and its AMBER instrument, which combine high spatial resolution ( 2 mas for the $200 \mathrm{~m}$ baseline) and a spectral resolution of 10000 , the "direct" observation of a varying inner disk radius, certainly correlated to circumstellar outbursts, will be soon accessible. This kind of observation has already be done by Guilloteau et al. (1999) for GG Tau using the IRAM interferometer. We can see in Fig. 11 that the real part of the $1.4 \mathrm{~mm}$ continuum visibility as a function of the IRAM baseline length (dots with error bars) agrees very well with a model

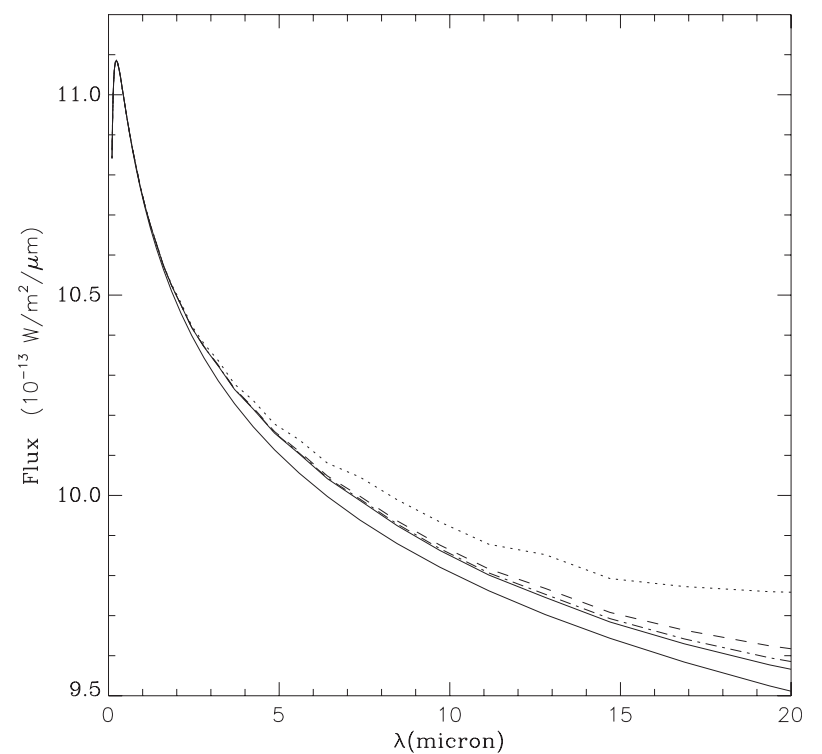

Fig. 8. Spectral energy distribution (SED) as a function of the mass loss rate: only the central star (lower plain line), $4.7 \times 10^{-10}$ (dotted-line), $2.3 \times 10^{-10}$ (dashed-line), $9.4 \times 10^{-11}$ (dash-dot-line) and $4.7 \times 10^{-11}$ (dash-dot-dot line) $M_{\odot}$ year $^{-1}$. Since the disk is nearly optically thin, the SED is nearly independent of the inclination angle between the star rotation axis and the observer's line of sight.

(plain line) where $90 \%$ of the flux is coming from a circumbinary ring and the rest from an extended disk. The global shape of the visibility curve is globally the same as for our pole-on ring models from Fig. 3 even if we have plotted visibilities modulus (between 0 and 1) in our modeling whereas this is the real part of 

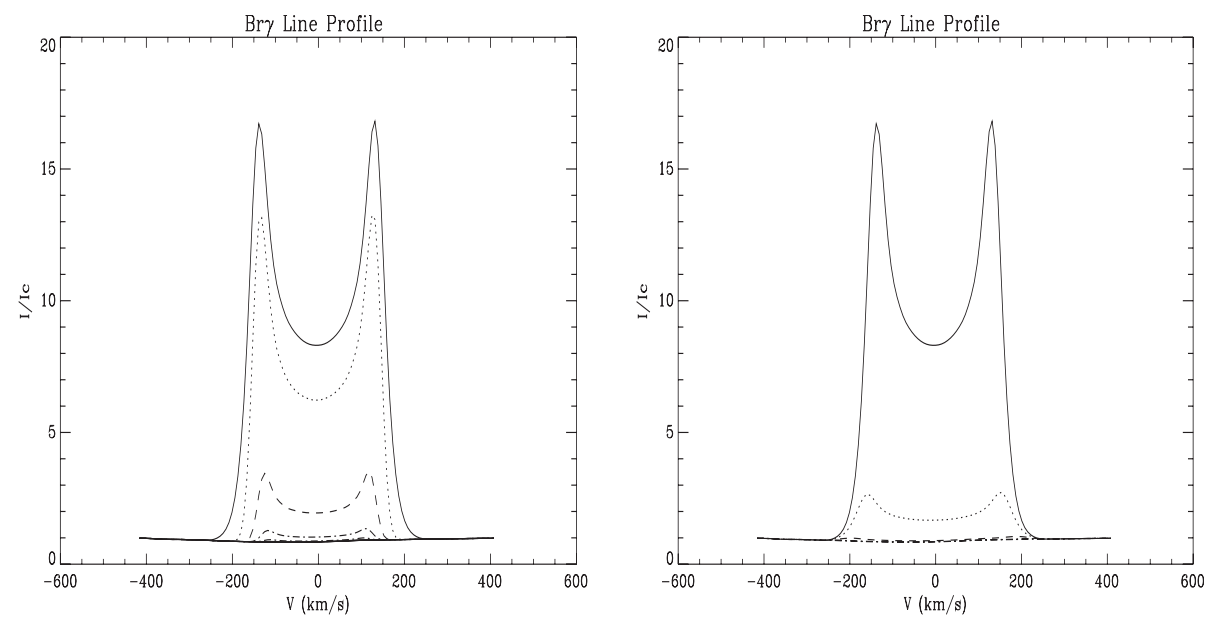

Fig. 9. Br $\gamma$ line profiles from the ring (left) and mass flux scenarios computed for an inclination angle of $45^{\circ}$ between the star rotation axis and the observer's line of sight as a function of the disk dissipation.
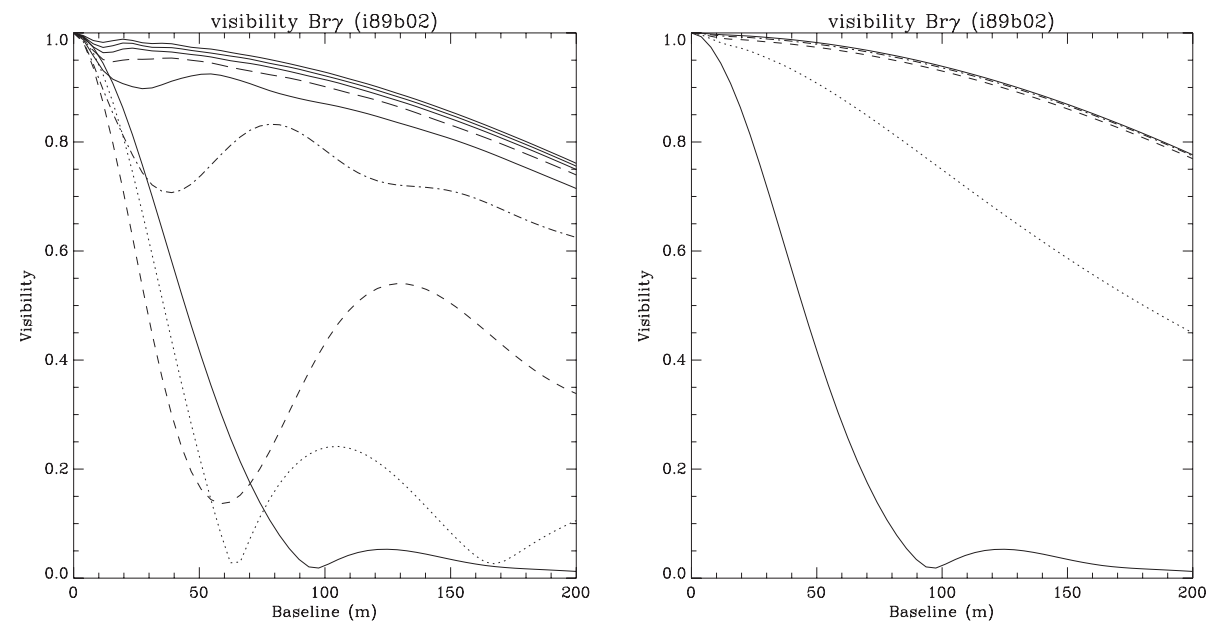

Fig. 10. Visibilities as a function of the baseline length for a baseline orientation along the equatorial disk as a function of the disk dissipation: following the ring model (left) and the decreasing mass flux scenario (right).

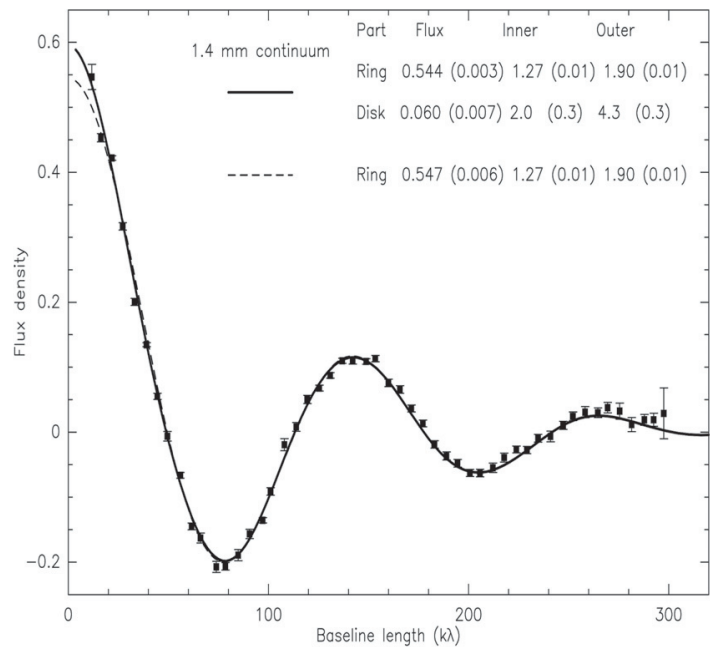

Fig. 11. GG Tauri real part of the $1.4 \mathrm{~mm}$ continuum visibility as a function of the IRAM baseline length (dots with error bars) with a model (plain line) where $90 \%$ of the flux comes from a ring and the rest from an extended disk from Fig. 2 in Guilloteau et al. (1999). Note that this is the real part of the visibility (which can be negative!) whereas in our simulation we plotted the visibility modulus that is always between 0 and 1 . The global shape of the visibility curve is the same as for our ring model from Fig. 3 seen pole-on. the visibility, which can be negative, that is plotted in Guilloteau et al. (1999).

Acknowledgements. We thank Anne Dutrey and Stéphane Guilloteau for providing and allowing us to use their Fig. 2 in this paper. We also thank Damien Mattei for his help in developing the SIMECA code, as well as David Chapeau, Jean-Michel Clausse and Pascal Marteau for their support in the computer and network settings. J. R. R. Tolkien is also acknowledged for helping us to find the right title for this paper.

\section{References}

Arias, M. L., Zorec, J., Cidale, L. S., \& Ringuelet, A. E. 2006, A\&A, submitted Araújo, F. X., \& Freitas Pacheco, J. A. 1989, MNRAS, 241, 543

Arthur, S. J., Dyson, J. E., \& Hartquist, T. W. 1994, MNRAS, 269, 1117

Bjorkman, J. E., \& Cassinelli, J. P. 1993, ApJ, 409, 429

Brown, J. C., \& Wood, K. 1992, A\&A, 265, 663

Castor, J. I., Abbott, D. C., \& Klein, R. I. 1975, ApJ, 195, 157

Chen, H., Marlborough, J. M., \& Waters, L. B. F. M. 1992, ApJ, 384, 605

Clark, J. S., Tarasov, A. E., \& Panko, E. A. 2003, A\&A, 403, 239

Cook, K. H., Alcock, C., Bennett, D. P., et al. 1995, in Astrophysical Applications of Stellar Pulsations, IAU Coll., 155, ed. R. S. Stobie, \& P. A. Whitelock, ASP Conf. Ser., 83, 221

Dyson, J. E., \& Hartquist, T. W. 1992, Astro. Lett. Comm., 28, 301

Floquet, M., Hubert, A. M., Hirata, R., et al. 2000, A\&A, 362, 1020

Frémat, Y., Zorec, J., Hubert, A. M., \& Floquet, M. 2005, A\&A, 440, 305

Grady, C. A., Bjorkman, K. S., \& Snow, T. P. 1987, ApJ, 320, 376

Grady, C. A., Bjorkman, K. S., Snow, T. P., et al. 1989, ApJ, 339, 403

Guilloteau, S., Dutrey, A., \& Simon, M. 1999, A\&A, 348, 570 
Guinan, E. F., \& Hayes, D. P. 1984, ApJ, 287, L39

Hanuschik, R. W. 1995, A\&A, 295, 423

Hanuschik, R. W. 1996, A\&A, 308, 170

Hanuschik, R. W., Dachs, J., \& Baudzus, M., et al. 1993, A\&A, 274, 356

Hartquist, T. W., Dyson, J. E., Pettini, M., et al. 1986, MNRAS, 221, 715

Hubeny, I. 1988, Comp. Phys. Comm., 52, 103

Hubeny, I., \& Lanz, T. 1995, ApJ, 439, 875

Hubert, A. M., \& Floquet, M. 1998, A\&A, 335, 565

Hubert, A. M., Floquet, M., \& Zorec, J. 2000, in The Be phenomenon in earlytype stars, IAU Coll., 175, ed. M. A. Smith, H. F. Henrichs, \& J. Fabregat, ASP Conf. Ser., 214, 348

Hummel, W. 1994, A\&A, 289, 458

Hummel, W., \& Dachs, J. 1992, A\&A, 262, L17

Kastner, J. H., \& Mazzali, P. A. 1989, A\&A, 210, 295

Keller, S. C., Bessell, M. S., Cook, K. H., Geha, M., \& Syphers, D. 2002, AJ, 124, 2039

Kwok, S., Purton, C. R., \& FitzGerald, P. M. 1978, ApJ, 219, L125

Lee, U., Saio, H., \& Osaki, Y. 1991, MNRAS, 250, 432

Limber, D. N. 1964, ApJ, 140, 1391

Limber, D. N. 1967, ApJ, 148, 141

Limber, D. N. 1969, ApJ, 157, 785

Marlborough, J. M. 1969, ApJ, 156, 135

Mennickent, R. E., Pietrzyński, G., Gieren, W., \& Szewczyk, O. 2002, A\&A, 393,887

Moujtahid, A., Zorec, J., \& Hubert, A. M. 1998, A\&AS, 129, 289

Moujtahid, A., Zorec, J., \& Hubert, A. M. 1999, A\&A, 349, 151

Okazaki, A. T. 2001, PASJ, 53, 119

Owocki, S. P., Gayley, K., \& Cranmer, S. 1996, ApJ, 472, L115

Pavlovski, K., Harmanec, P., Božič, H., et al. 1997, A\&AS, 125, 75

Percy, J. R., Harlow, J., Hayhoe, K. A. S., et al. 1997, PASP, 109, 1215

Pikel'ner, S. B. 1968, Astrophys. Lett., 2, 97

Poe, C. H., \& Friend, D. B. 1986, ApJ, 311, 317
Poeckert, R., \& Marlborough, J. M. 1987a, ApJ, 220, 940

Poeckert, R., \& Marlborough, J. M. 1987b, ApJS, 38, 229

Porter, J. M. 1997, A\&A, 324, 597

Porter, J. M. 1999, A\&A, 348, 512

Quirrenbach, A., Bjorkman, K. S., Bjorkman, J. E., et al. 1997, ApJ, 479, 477

Rivinus, Th., Baade, D., Stefl, S., \& Maintz, M. 2001, A\&A, 379, 257

Rohrmann, R. D. 1997, MNRAS, 285, 427

Snow, T. P. 1982, ApJ, 253, L39

Stee, Ph. 2003, A\&A, 403, 1023

Stee, Ph., \& Araújo, F. X. 1994, A\&A, 292, 221

Stee, Ph., \& Bittar, J. 2001, A\&A, 367, 532

Stee, Ph., Araújo, F. X., Vakili, F., et al. 1995, A\&A, 300, 219

Stee, Ph., Vakili, Bonneau, D., et al. 1998, A\&A, 332, 268

Schmitz, F. 1983, A\&A, 120, 234

Struve, O. 1931, ApJ, 73, 94

Struve, O. 1942, ApJ, 95, 134

Thomas, R. N., \& Doazan, V. 1982, in B Stars With and Without

Tycner, C., Lester, J. B., Hajian, A. R., et al. 2005, ApJ, 624, 359

Vinicius, M. M. F., Zorec, J., Leister, N. V., \& Levenhagen, R. S. 2006, A\&A, 446, 643

Waters, L. B. F. M. 1986, A\&A, 162, 121

Waters, L. B. F. M., Coté, J., \& Lamers, H. J. G. L. M. 1987, A\&A, 185, 206

Wood, K., Bjorkman, K. S., \& Bjorkman, J. E. 1997, ApJ, 477, 926

Zorec, J. 1981, in Effects of Mass-Loss in Stellar Evolution, ed. C. Chiosi, \& R. Stalio, IAU Coll., 59, 539

Zorec, J., Frémat, Y., \& Hubert, A. M. 2000a, in The Be phenomenon in earlytype stars, IAU Coll., 175, ed. M. A. Smith, H. F. Henrichs, \& J. Fabregat, ASP Conf. Ser., 214, 330

Zorec, J., Hubert, A. M., \& Moujtahid, A. 2000b, in The Be phenomenon in early-type stars, IAU Coll., 175, ed. M. A. Smith, H. F. Henrichs, \& J. Fabregat, ASP Conf. Ser., 214, 448 